\title{
WAVELET TRANSFORM IN IMAGE RECOGNITION
}

\author{
Aleš Procházka, Andrea Gavlasová, and Karel Volka \\ Prague Institute of Chemical Technology \\ Technická 1905, 16628 Prague 6, Czech Republic \\ E-mail: A.Prochazka@ieee.org
}

\begin{abstract}
Texture segmentation and classification form a very important topic of the interdisciplinary area of signal and image processing with many applications in satellite image processing, biomedical image analysis, environmental engineering and microscopic image processing. The paper presents selected mathematical methods used for image segmentation and application of wavelet transform for the following segments classification by multiresolution decomposition of segments boundary signals. The wavelet transform approach has been adopted here and used for feature extraction allowing its use for image denoising and resolution enhancement as well. Results of feature extraction obtained by the discrete wavelet transform are compared with that obtained by the discrete Fourier transform. Feature classification is then achieved by self-organizing neural networks. Proposed methods has been verified for simulated structures and then used for analysis of microscopic images of crystals of different shape and size.
\end{abstract}

\section{INTRODUCTION}

A basic problem encountered in the digital image processing is in image de-noising $[12,16$, 17], restoration of its missing or corrupted components $[9,7]$ and its enhancement [14], feature extraction [13] and classification [8].

The paper is devoted the use of wavelet transform and multiresolution image decomposition for image segmentation and classification $[2,1,5,11,9]$. An example the application of proposed methods in crystalography is presented in Fig. 1 showing the microscopic image of crystals of different shape, texture and size. Similar methods can be also used in other ares including biomedical imaging, environmental image processing or analysis of satellite observations.

Image components detection [3, 15], segmentation and feature extraction can then be followed by their classification. The paper prese: a proposed method using self-organizing neu networks [4] and class boundary estimation. methods are verified in the Matlab environme

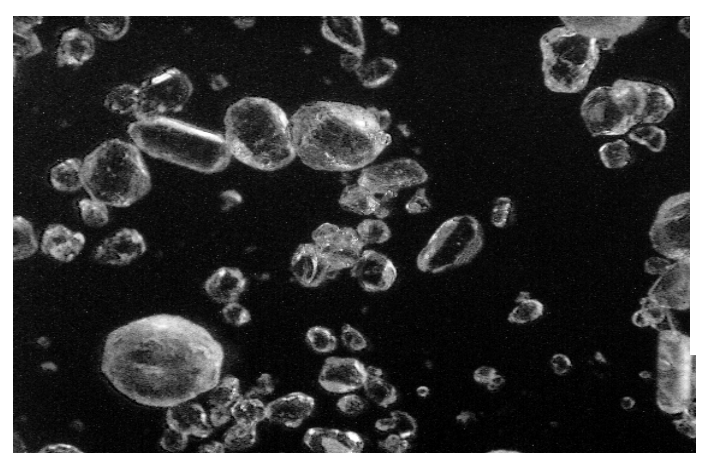

Fig. 1. Microscopic image of crystals of different shape, texture and size

\section{IMAGE SEGMENTATION}

Image segmentation represents an important initial step of image processing. Fig. 2(a) presents an example of a simulated image containing segments of different shapes and textures and its segments extraction using the watershed method [8]. The proposed algorithm consists of these steps

- image thresholding to convert it to the black and white form

- distance and watershed transform use to find image ridge lines presented in Fig. 2(b)

- extraction of a segment and its boundary presented in Fig. 2(c)

- area texture extraction presented in Fig. 2(d) Principle of image segmentation and boundary signal definition is presented in Fig. 3.

The classification process assumes definition of a pattern matrix containing features of sepa-

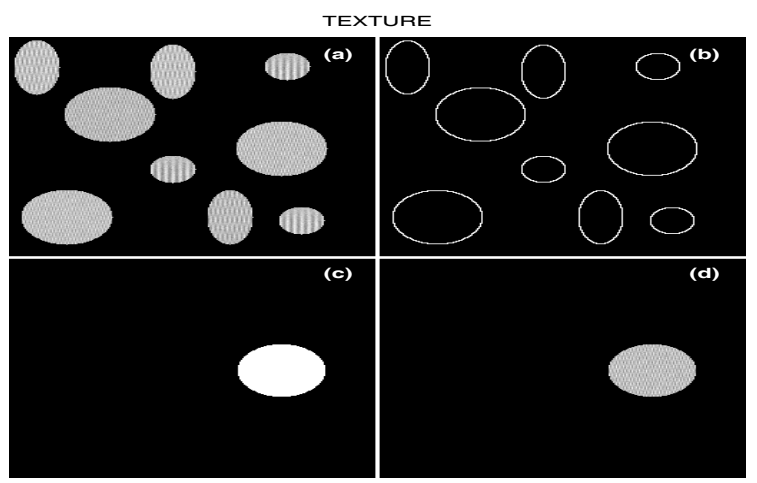

Fig. 2. Image segmentation presenting (a) an image containing different simulated structures, (b) results of its watershed segmentation, (c) selected image segment area, and (d) its texture 


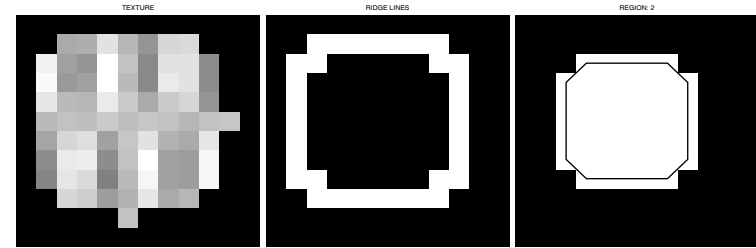

Fig. 3. Principle of image segmentation and image boundary selection

rate image segments. Many possibilities of their extraction [13] include analysis of

- image boundary signal or the texture inside its area

- statistical properties of boundary signal or segment structure

- transform of boundary signal or segment structure allowing its translation independence and using discrete wavelet transform or discrete wavelet transform among others

Fig. 4 presents the boundary signal of a segment of Fig. 2 in (a) three or (b) two dimensions, and (c) its discrete Fourier transform. Image features used for their classification can be based upon the mean value and the variance of discrete Fourier transform coefficients. Wavelet transform discussed further can be even more efficient allowing multiresolution signal or image analysis.

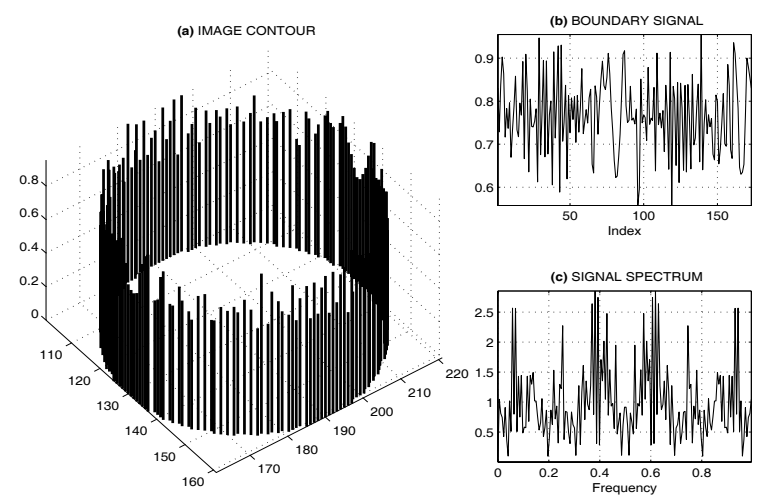

Fig. 4. Selected image segment analysis presenting (a) three dimensional image segment boundary signal, (b) two dimensional image boundary signal, and

(c) its discrete Fourier transform

\section{IMAGE WAVELET DECOMPOSITION AND RECONSTRUCTION}

Signal wavelet decomposition using Discrete Wavelet Transform (DWT) provides an alternative to the Discrete Fourier Transform (DFT) for signal analysis resulting in signal decomposition into two-dimensional functions of time and scale. The main benefit of DWT over DFT is in its multi-resolution time-scale analysis ability.

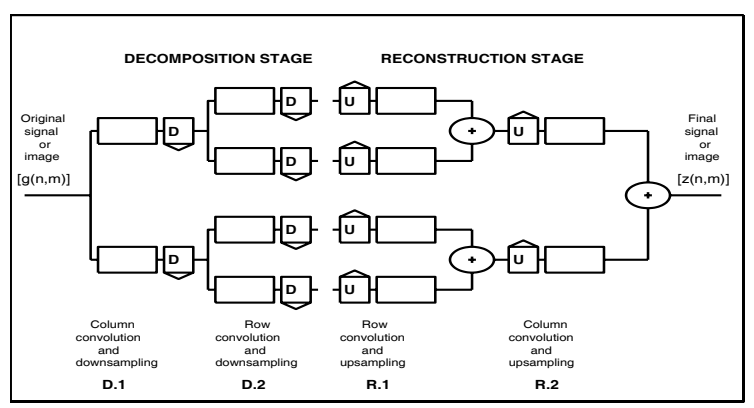

Fig. 5. Wavelet transform use in image decomposition and reconstruction

Wavelet functions used for signal analysis are derived from the initial function $W(t)$ forming basis for the set of functions

$$
W_{m, k}(t)=\frac{1}{\sqrt{a}} W\left(\frac{1}{a}(t-b)\right)
$$

for discrete parameters of dilation $a=2^{m}$ and translation $b=k 2^{m}$. Wavelet dilation, which is closely related to spectrum compression, enables local and global signal analysis.

The principle of signal and image wavelet decomposition and reconstruction $[10,14]$ is presented in Fig. 5 for an image matrix $[g(n, m)]_{N, M}$ or one-dimensional signal $x[n]_{N, 1}=[g(n, 1)]_{N, 1}$ considered as a special case of an image having one column only. The highpass filter and the complementary lowpass filter is applied to image columns and then to its rows followed by downsampling after each processing unit at first. Resulting coefficients can be used for image or signal analysis or for its reconstruction again. Fig. 6 presents wavelet decomposition of a selected image segment boundary signal.
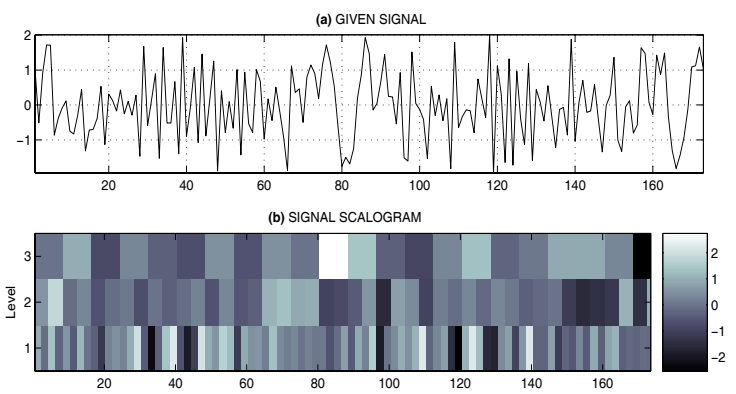

(c) SIGNAL SCALING AND WAVELET COEFFIIIENTS: LEVELS 1-3

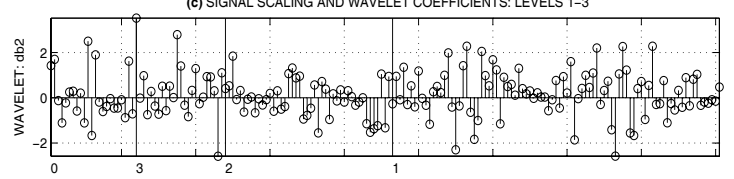

Fig. 6. Selected image segment analysis presenting (a) two dimensional image segment boundary signal, (b) scalogram presenting coefficients of its decomposition into three levels, and (c) wavelet transform coefficients organized in a row vector 
Studying the first decomposition step let us denote a column of the image matrix $[g(n, m)]_{N, M}$ as signal $\{x(n)\}_{n=0}^{N-1}=[x(0), x(1), \ldots, x(N-1)]^{\prime}$. This signal can be analyzed by a half-band lowpass filter with its impulse response

$$
\{s(n)\}_{n=0}^{L-1}=[s(0), s(1), \cdots, s(L-1)]
$$

and corresponding high-pass filter based upon impulse response

$$
\{w(n)\}_{n=0}^{L-1}=[w(0), w(1), \cdots, w(L-1)]
$$

The first stage assumes the convolution of a given signal and the appropriate filter for decomposition at first by relations

$$
\begin{aligned}
x l(n) & =\sum_{k=0}^{L-1} s(k) x(n-k) \\
x h(n) & =\sum_{k=0}^{L-1} w(k) x(n-k)
\end{aligned}
$$

for all values of $n$ followed by subsampling by factor $D$. In the following decomposition stage $D .2$ the same process is applied to rows of the image matrix followed by row downsampling. The decomposition stage results in this way in four images representing all combinations of low-pass and high-pass initial image matrix processing.

The reconstruction stage includes row upsampling by factor $U$ at first and row convolution in stage $R .1$. The corresponding images are then summed. The final step $R .2$ assumes column upsampling and convolution with reconstruction filters followed by summation of the results again.

In the case of one-dimensional signal processing, steps D.2 and R.1 are omitted. The whole process can be used for signal or image

(1) decomposition and perfect reconstruction allowing signal or image compression

(2) resolution enhancement and interpolation for reconstruction of corrupted image regions

(3) feature extraction using the variance of summed squared coefficients at a selected level of decomposition

(4) de-noising based upon modification of image decomposition coefficients using selected threshold limits

In all these cases the multiresolution properties of wavelet transform is used. Fig. 7 presents this property for a selected wavelet function.
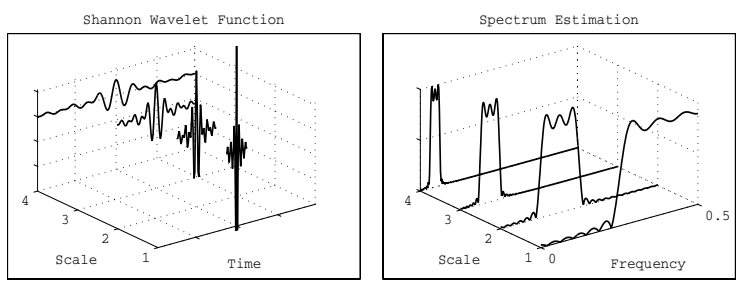

Fig. 7. Shanon wavelet and the effect of its dilation to spectrum compression

\section{IMAGE REGIONS CLASSIFICATION}

Classification of $Q$ images using $R$ features organized in pattern matrix $\mathbf{P}_{R, Q}$ can be realized by application of self-organizing neural networks. The number $S$ of output layer elements is equal to image classes and must be either defined in advance or it can be automatically increased to create new classes by special methods [6]. During the learning process network weights forming matrix $\mathbf{W}_{S, R}$ are changed to minimize distances between each input vector and corresponding weights of a winning neuron characterized by its coefficients closest to the current pattern. In case that the learning process is successfully completed network weights belonging to separate output elements represent typical class individuals.

Class boundaries presented in Fig. 8 are defined by values of matrix $\mathbf{W}_{S, R}$ and for $R=2$ have been evaluated by a proposed algorithm including

- evaluation of distances of variables belonging to the first and second feature and output neuron weights

- definition of potential boundary lines making equal individual distances

- calculation of intersection points for each line

- selection of proper boundary segments dividing the plane into the number of regions equal to classes

Results of classification of image segments given in Fig. 2(a) into three classes are presented in Fig. 8 for two selected signal features obtained (i) as the mean and variance of discrete Fourier transform (DFT) coefficients and (ii) by the variance of discrete wavelet transform (DWT) coefficients at the first and the second level of boundary signal values. Results obtained by both methods are the same but the variance of features evaluated by the DWT is smaller comparing to that obtained by the DFT as presented in Table 1.
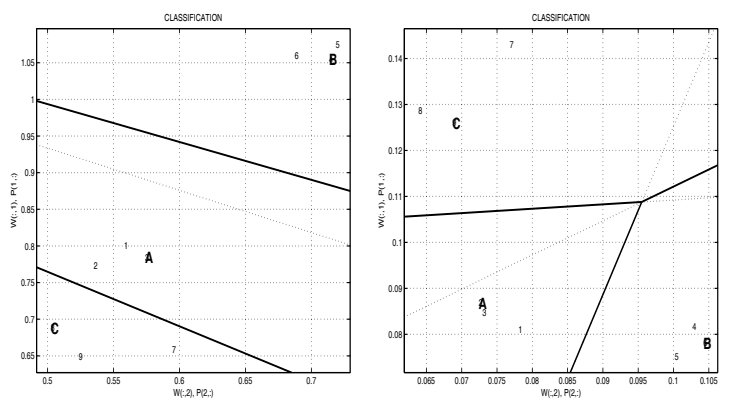

Fig. 8. The topography of features resulting from (a) the discrete Fourier transform and (b) the discrete wavelet transform for the simulated image and their classification into three classes providing class boundaries as well 
Table 1. Comparison of image segments classification into three classes using two features evaluated by DFT and DWT using DB4 wavelet function

\begin{tabular}{|l|ccc|}
\hline \multirow{3}{*}{ Feature } & \multicolumn{3}{|c|}{ Typical Class Image } \\
\cline { 2 - 4 } & \multicolumn{3}{|c|}{ Class Mean Square Errors } \\
\cline { 2 - 4 } & $A$ & $B$ & $C$ \\
\hline \hline DFT & 3 & 4 & 8 \\
& 0.020 & 0.014 & 0.048 \\
\hline DWT & 2 & 6 & 9 \\
& 0.004 & 0.003 & 0.010 \\
\hline
\end{tabular}

\section{RESULTS}

Results of a selected part of the real image representing the microscopic image of crystals is presented in Fig. 9. Image segmentation using watershed transform is able to detect most of image segments even though the problem of fault class boundaries can arise in some cases.

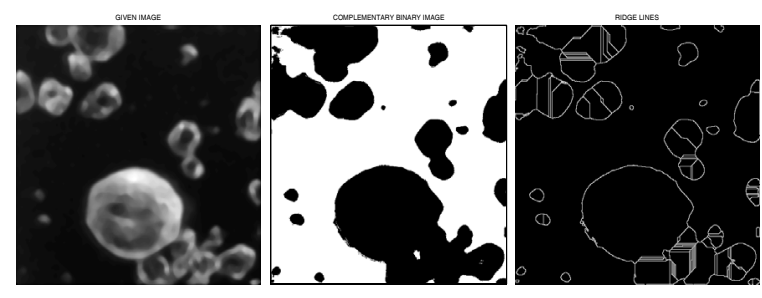

Fig. 9. Real image segmentation results

\section{CONCLUSION}

The paper presents some possibilities of image segmentation and classification using wavelet transform. It is possible to summarize that wavelet transform provide many possibilities of detection of image segment features owing to its multiresolution properties and the possibility to choose different wavelet functions appropriate for a given problem as well. Segments boundary signals were used for image classification even though there it is possible to use two dimensional wavelet transform for detection of patterns of individual segments texture, too.

Methods discussed in the paper has been applied to analysis of shapes of microscopic images of crystals. Similar methods can be used in other applications in a wide range of interdisciplinary problems of texture analysis including biomedical imaging, processing of satellite images, communications and remote earth observations.

\section{ACKNOWLEDGMENTS}

The work has been supported by the research grant of the Faculty of Chemical Engineering of the Institute of Chemical Technology, Prague No. MSM 223400007.

\section{REFERENCES}

[1] S. Arivazhagan and L. Ganesan. Texture classification using wavelet transform. Pattern Recogn. Lett., 24(9-10):1513-1521, 2003.

[2] S. Arivazhagan and L. Ganesan. Texture segmentation using wavelet transform. Pattern Recogn. Lett., 24(16):3197-3203, 2003.

[3] S. Arivazhagan and L. Ganesan. Automatic target detection using wavelet transform. Eurasip Journal on Applied Sig. Proc., 17:2663-2674, 2004.

[4] C. M. Bishop. Neural Networks for Pattern Recognition. Oxford University Press, 1995.

[5] A. Bruce, D. Donoho, and Hong-Ye Gao. Wavelet Analysis. IEEE Spectrum, 33(10):26-35, October 1996.

[6] D. I. Choi and S. H. Park. Self-Creating and Organizing Neural Networks. IEEE Trans. Neural Networks, 5(4):561-575, July 1994.

[7] Alessandro Duci, Anthony J. Yezzi, Sanjoy K. Mitter, and Stefano Soatto. Region matching with missing parts. In $E C C V$ (3), pages 48-64, 2002.

[8] R. C. Gonzales, R. E. Woods, and S. L. Eddins. Digital Image Processing Using MATLAB. Prentice Hall, 2004.

[9] Onur G. Guleryuz. Iterated Denoising for Image Recovery. In Data Compression Conference (DCC '02), Snao Bird, Utah. IEEE, 2002.

[10] N. G. Kingsbury. Complex Wavelets for Shift Invariant Analysis and Filtering of Signals. Journal of Applied and Computational Harmonic Analysis, 10(3):234-253, May 2001.

[11] S. Li and J. Shawe-Taylor. Comparison and fusion of multiresolution features for texture classification. Pattern Recogn. Lett., 25, 2004.

[12] D. E. Newland. An Introduction to Random Vibrations, Spectral and Wavelet Analysis. Longman Scientific \& Technical, Essex, U.K., third edition, 1994.

[13] M. Nixon and A. Aguado. Feature Extraction 65 Image Processing. NewNes Elsevier, 2004.

[14] J. Ptáček, I. Šindelářová, A. Procházka, and J. Smith. Wavelet Transforms In Signal and Image Resolution Enhancement. In Int. Conf. Algoritmy02, Podbanske. STU, 2002.

[15] T. Randen and J. H. Husoy. Filtering for Texture Classification: A Comparative Study. IEEE Trans. on PAMI, 21(4):291310, 2000.

[16] G. Strang. Wavelets and Dilation Equations: A brief introduction. SIAM Review, 31(4):614-627, December 1989.

[17] Saeed V. Vaseghi. Advanced Signal Processing and Digital Noise Reduction. Wiley \& Teubner, West Sussex, U.K., 2000. 